\title{
Comportamiento y experiencia de consumo desde la interconexión e interactividad de la World Wide Web: un recorrido teórico
}

Behavior and experience of consumption from interconnectivity and interactivity in the World Wide

Web: a theoretical review feasibility

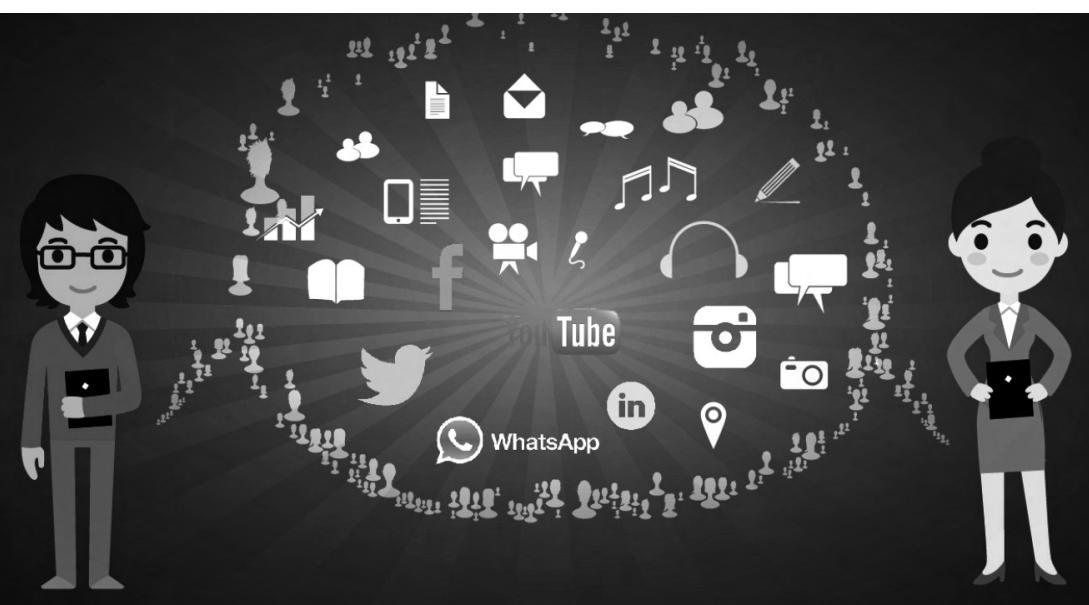

rma

$r:$

ZyB

OLj

AuM $\mathrm{Gg}+$ $\mathrm{Niw}$ $\mathrm{XMS}$ $w \times N$ $-4 x$

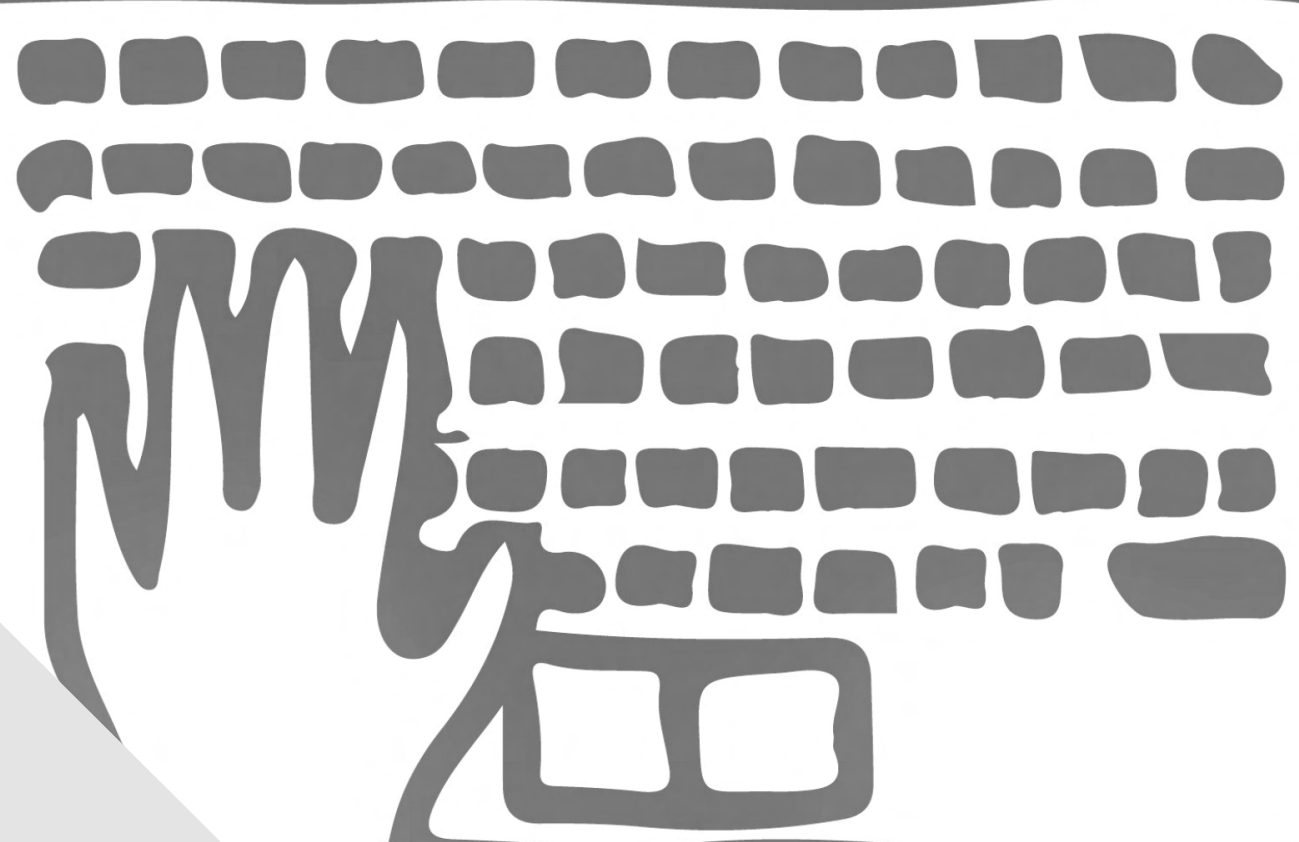

11 , sy 51 , em0, sy $306, \ldots 4 \mathrm{v}$

po ding:

$11 \mathrm{px} ;$ WdodD0 iM weCIgd2 -DI5LjI1 iBDNi42 IsMi UNjAxIDIS i000 XNC 42N j UsNy400 rogy'MS42 j I1MywyMi4wMSAxMS OTUgMTMuNTIyLDEXL. 1LDE4Lj kzNSAxNC43i AxNC42NzgsOC43Nj 44NzIsMTcuNiA5Lj jYyMiA2LjM4NywXN OTEgQzIzLjA30CWX 2NDc sMjMuMDUgMTI M3IEMyMS45NSwyNy4_ PranPiwvr 37nPn=="1) 


\title{
Comportamiento y experiencia de consumo desde la inter- conexión e interactividad de la World Wide Web: un recorri- do teórico ${ }^{1}$
}

\section{Behavior and experience of consumption from interconnectivity and interactivity in the World Wide Web: a theoretical review}

\author{
Ignacio Osuna Soto ${ }^{2}$, Cindy Paola Pinzón Rios ${ }^{3}$ \\ Inalde Business School, Universidad de La Sabana, Chía, Colombia
}

Artículo recibido en abril de 2106; artículo aceptado en junio de 2016

\begin{abstract}
Citación del artículo: Osuna, I. \& Pinzón, C. (2016). Comportamiento y experiencia de consumo desde la interconexión e interactividad de la World Wide Web: un recorrido teórico. I+D Revista de Investigaciones, 8 (2), pp. 35 - 45
\end{abstract}

\begin{abstract}
Resumen
La evolución de la World Wide Web ha generado una mayor interconexión entre usuarios propiciando el análisis de su comportamiento a partir del tipo de interacción y desarrollando un mundo en línea homólogo al que no lo está (off line), que reta a las empresas a intensificar las experiencias de consumo y atraer la atención de los consumidores. Para indagar sobre los principales aportes y cambios en la forma de relacionarse socialmente desde la web, el artículo hace un recorrido teórico en el que se evidencia que el acceso a data implica un reto para las empresas que buscan comprender a fondo lo que realmente quieren sus clientes y las posibles formas de retenerlos, para vincularlos con el valor de la marca mediante canales de comunicación que rompan lo tradicional.
\end{abstract}

Palabras clave: web 3.0, redes sociales, análisis de comportamiento, ciencia cognitiva, inteligencia artificial.

\begin{abstract}
The evolution of the World Wide Web has generated a great interconnection between users, which enables a behavioural analysis, developing an on-line world homologous to the off-line world one which challenges companies to intensify the consumer experiences and to attract customers' attention. The objective of this theoretical review is to explain the contributions and changes to the way we socialize though the web, evidencing that the access to data implies a challenge for companies to understand thoroughly what customers really want, the possible ways to retain and link them with the brand value through communication channels that disrupt the traditional communications.
\end{abstract}

Key words: web 3.0, social media, behaviour analysis, cognitive science, artificial intelligence.

1. Artículo de revisión teórica perteneciente a la Dirección Comercial y de Operaciones, desarrollado en el grupo de investigación Empresa, competitividad y marketing, y financiado por el Inalde Business School, Universidad de La Sabana, Chía, Colombia. Dirección: Autopista Norte Km

7 Costado Occidental, Puente del Común, Chía, Cundinamarca, Tel: 8614444. Fecha: 2016

2. Ingeniero industrial, Universidad de La Sabana. Ph.D. in Management (Marketing), IESE Business School (España). Profesor-investigador del grupo Empresa, competitividad y marketing, INALDE Business School, Universidad de La Sabana, Chía, Colombia: Dirección: Autopista Norte Km 7 Costado Occidental, Puente del Común, Chía, Cundinamarca, Tel.8614444. ignacio.osuna@inalde.edu.co

3. Comunicadora social y periodista, y estudiante de décimo semestre de Psicología, Universidad de La Sabana. Investigadora del grupo Empresa, competitividad y marketing, Inalde Business School, Universidad de La Sabana, Chía, Colombia: Dirección: Autopista Norte Km 7 Costado

Occidental, Puente del Común, Chía, Cundinamarca, Tel. 8614444. cindy.pinzon@inalde.edu.co 
La red informática mundial o World Wide Web (en adelante WWW) es una plataforma que busca facilitarle a los usuarios el intercambio de información mediante una base tecnológica en la cual hay contenido generado por el propio usuario; dicha base es accesible públicamente en un sitio web, que a su vez implica un esfuerzo creativo con una intención de mercadeo (Kaplan \& Haenlein, 2010). Por medio de esta plataforma se interconecta la información entre usuarios, los cuales pueden expresar sus opiniones desde diferentes plataformas y tipos de estructuras (Rahwan, Zablith \& Reed, 2007). En la WWW se encuentra el Social Media o redes sociales, que permiten al usuario crear e intercambiar contenido mediante diferentes herramientas y servicios como Facebook, YouTube y Twitter, entre otras, que tienen como base elementos de presencia social enmarcados por la auto-presentación y la autorrevelación (Kaplan et al., 2010).

Las redes sociales se caracterizan por funciones como la identificación, el relacionamiento, construir reputación, la comunicación, y el reconocimiento propio y de los demás, al estar conformada por grupos interconectados y por el intercambio de información (Kietzmann, Hermkens, McCarthy \& Silvestre, 2011). Las personas hacen uso de las redes sociales por diferentes aspectos motivacionales y actitudinales como esparcimiento, diversión, interés de hacer contactos y por los efectos sociales; también porque si tienen alguna inclinación por los sistemas de información, encuentran la información que necesi$\tan ,($ Lin \& Lu, 2011).

A partir de la web y las redes sociales digitales (redes sociales en adelante) se generan vínculos que permiten compartir los intereses y expresar la identidad de las personas a través del uso de diferentes dispositivos que median la comunicación, cuya evolución en la web se inicia con la web 1.0, caracterizada por el PC, seguida de la web 2.0, conocida por la integración con dispositivos móviles, que, a su vez, traza el camino hacia la web 3.0, en la cual se incrementa el nivel de interacción digital e interconexión, propiciando un mayor acceso a la data (Hall \& Tiropanis, 2012). De esta manera, la WWW ha implicado un cambio en el planteamiento de las empresas hacia los consumidores, revolucionando los negocios, al igual que la forma de estrechar y crear vínculos con los clientes, lo cual implica un desafío para los gerentes de mercadeo, debido a que deben crear valor compartido con los consumidores, reconocer sus hábitos y tener una reacción inmediata frente a sus necesidades (Berthon, Pitt, Plangger \& Shapiro, 2012).

Al contar con un flujo de información e interacción mayor entre los usuarios, las empresas pueden conocer y acercarse a los consumidores para generar una mejor segmentación, entender las necesidades e intensificar la experiencia con la marca, incrementar el nivel de interconexión e interacción, creando valor en los consumidores y construyendo relaciones (Michaelidou, Siamagka \& Christodoulides, 2011). Frente a este panorama, se hace una revisión teórica para indagar por la forma en que, por medio de la interconexión e interacción en la WWW, se puede conocer el comportamiento de los consumidores e incrementar su experiencia.

\section{Web 2.0}

En la red hay un alto flujo de información que es almacenada y procesada por sistemas de búsqueda escalables, siendo Google el principal buscador de occidente, el cual, a partir de algoritmos, recopila las páginas web, las indexa para calificar la calidad, el anclaje con el texto y la información por proximidad, generando así un sistema de búsqueda de información que clasifica aproximadamente 24 millones de páginas semanalmente, con una capacidad de construir índices de 100 millones de páginas en un mes (Brin \& Page, 2012). Baidu es el servicio de indexación análogo en China, donde Google no presta su servicio. La existencia de este buscador prueba el significado del servicio en el desarrollo, uso e interacción de la WWW con el consumidor.

Sin embargo, no toda la información existente en la web es procesada en su totalidad por los usuarios, de hecho el $90 \%$ de los usuarios usualmente solo ve las tres primeras páginas de los resultados (Sen, 2005). Esto implica que para conseguir una visibilidad al momento de realizar una búsqueda se necesita de una buena indexación en un ranking que esté en un nivel superior a los otros sitios web que pueden ser considerados como competencia, para lo cual es necesario generar un mayor tráfico, visibilidad y usabilidad del sitio web al incrementar su frecuencia de ingreso (Zuze \& Weideman, 2013).

Se ha establecido que una de las variables medidas por los algoritmos de los sistemas de búsquedas es la combinación de palabras o términos que describen a la marca o el producto, que son conocidas como palabras claves (key words), y que tienen la función de ser propaganda y de facilitar la búsqueda (Yang \& Ghose, 2010). De esta manera, se puede conseguir un buen posicionamiento del sitio web por medio de búsquedas orgánicas (Search Engine Optimization o SEO) o pagadas (Search Engine Marketing o SEM), estando las búsquedas orgánicas asociadas con una mayor probabilidad de generar clic a través de una búsqueda pagada (Yang \& Ghose, 2010).

En el posicionamiento mediante el SEO se debe tener en cuenta el uso de vínculos internos al igual que de pala- 
bras clave, que deben ser repetidas a lo largo de la página (Wilson \& Pettijohn, 2008) al ser un parámetro de indexación con una densidad esperada de entre el 3 y el $7 \%$; de ser mayor a este porcentaje el sitio web es penalizado (Zuze \& Weideman, 2013). Por otro lado, al garantizar el ingreso por un aproximado de diez días, el SEM permite estar entre las primeras opciones de búsqueda, para lo cual por Google AdWords se compran palabras clave relacionadas con el banner de publicidad (Sen, 2005).

\section{Interconexión en redes sociales}

En Colombia 8 de cada 10 personas acceden a internet diariamente y pasan cerca de 2,6 horas navegando, y 6 de cada 10 colombianos son usuarios de las redes sociales; de estos el $98 \%$ tiene cuenta en Facebook y el $31 \%$ en Twitter (Ministerio de Tecnologías de la Información y las Comunicaciones de Colombia, [Min TIC] 2014). Las personas que están activas en redes sociales como Facebook son más de un billón, de las cuales cien millones usan esta red mensualmente desde un dispositivo móvil (Facebook Newsroom, 2013) y los usuarios realizan más de cinco billones de actualizaciones en sus estados, los cuales están relacionados con sentimientos y actividades de todo tipo (Facebook Newsroom, 2014). Para el 2011 en Twitter había más de 175 millones de usuarios a nivel mundial, y las personas veían en promedio dos billones de videos en YouTube diariamente, siendo subidas veinticuatro horas de video cada minuto (Gupta, Armstrong \& Clayton, 2011).

En las redes sociales la interacción e intercambio de información es constante. En el caso de Facebook hay un flujo de información de aproximadamente 2,5 millones de terabytes, de los cuales solo se procesan diariamente 500; los usuarios de esta red social viven en una constante interacción, compartiendo cerca de 300 millones de fotografías diarias (Houghton, Joinson, Caldwell \& Marder, 2013). De esta forma, el acceso a la información que facilitan las redes sociales provee una serie de ventajas en el ámbito empresarial que hace unos años no se presentaban, como la posibilidad de conocer el comportamiento de los consumidores mediante insights y frecuencias de conductas reforzadas en el entorno social. Es así como se sabe que actualmente el $66 \%$ de los usuarios de Smartphone buscan información referente a comerciales vistos en televisión, al igual que el $82 \%$ de los usuarios realizan búsquedas cuando ven algún negocio local y el $91 \%$ buscan información mientras realizan alguna tarea alterna. Esto se ilustra en la Figura 1 (Think with Google, 2015, a).

La Figura 1 también muestra que el $82 \%$ de los usuarios miran sus Smartphones mientras están en un almacén decidiendo qué comprar; en YouTube, por su parte, entre las búsquedas más realizadas sobresale el "cómo hacer" (Think with Google, 2015, a). De hecho los millennials son unos buscadores sobresalientes de recetas de cocina por YouTube, siendo las búsquedas realizadas desde Smartphones referentes a "mejor receta", "ideas para la cena", "recetas saludables" y "recetas lentas para cocinar" el 59\% (Think with Google, 2015, b). Este hecho modifica la forma de interacción y de comunicación, debido a que el $57 \%$ de las personas mantienen más conversaciones en línea que en la vida real, y el $65 \%$ de los consumidores ven más información en línea respecto a otros años (Think with Google, 2015, a).

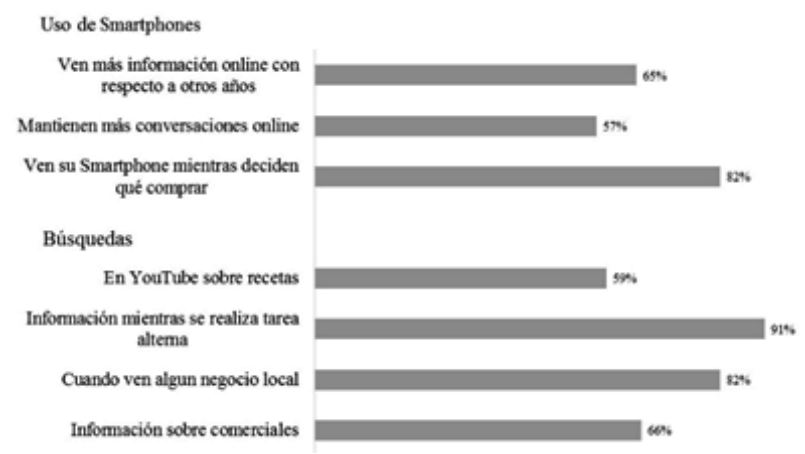

Figura 1.

Comportamiento en línea, (basado en Think with Google, 2015, a; Think with Google, 2015, b)

La información proporcionada por los usuarios en las redes sociales, como el reporte de lo que dicen y hacen frente a una marca o empresa, permite conocer patrones de conducta y esquemas cognitivos sociales que facilitan el establecimiento de estrategias publicitarias e informativas tendientes a modificar marcos relacionales, así como la percepción de la marca (Smith et al., 2008). También se puede conocer su comportamiento a partir de conductas de contagio social en las redes sociales, en donde hay una escala de tres personas de influencia frente a fenómenos como la felicidad, obesidad, cooperación y el tabaquismo (Christakis \& Fowler, 2013).

El comportamiento de los usuarios mantiene una relación e interconexión en grados de separación por nodos, que puede aumentar de 8 a 24 y 48 en redes aleatorias de poblaciones, en donde la información puede difundirse rápidamente, lo cual permite que se conecten nodos socialmente distantes para propagar acciones colectivas (Centola \& Macy, 2007). Una de las formas para identificar la relación existente entre los usuarios es mediante el Social Network Analysis (SNA), que también permite conocer la manera como la información se distribuye en el sistema de personas que conforman opiniones, símbolos y comportamientos que definen al grupo, desde la 
forma en que se comunican e interactúan (Burt, Kilduff \& Tasselli, 2013).

De esta manera, desde las redes sociales los mensajes relacionados con afectos positivos son asociados al compañerismo y a la satisfacción con la vida, debido a que implican un sentido de comunidad, al igual que un soporte social (Oh, Ozkaya \& LaRose, 2014). Lo anterior también se puede observar en fenómenos sociales en Facebook, en donde se extienden mensajes de esperanza, solidaridad y ayuda, por ejemplo, cuando el usuario poner como imagen de perfil la frase de "Pray for" o la bandera de un país que pasa por alguna dificultad, como ha ocurrido con Venezuela o Francia recientemente.

Esto implica la generación de fenómenos conocidos en redes sociales como virales, que evidencian la forma como los mensajes en línea pueden influenciar el comportamiento sin conexión, la búsqueda de información y la autoexpresión, tanto de la persona que recibe el mensaje como de su red de amigos (Bond et al., 2012). Otra forma de viralidad son los memes, caracterizados por tener un contenido de burla, enmarcado por mensajes irónicos que identifican esquemas cognitivos de una comunidad, explicando también su cultura y creencias, y guiando el comportamiento mediante mensajes simples, concretos, inesperados y emotivos con contenido político, económico y social (Rodríguez, 2013).

Algunas empresas han conseguido sacar provecho de la interconexión en redes sociales para promover ventas y estrechar su relación con los clientes; es el caso de Skoda, que en noviembre de 2011 realizó la campaña "Yo y mi Skoda" en donde, a través de Facebook, invitó a las personas a subir fotos con su Skoda (Houghton, et al., 2013). Por su parte, Davivienda, con la campaña "El Corresponsal" incrementó su visibilidad y recordación al aprovechar la coyuntura del Mundial de Fútbol del 2010 realizando pequeños cortos de un reportero que, al transmitir partidos de fútbol, atravesaba varias calamidades, durante las cuales hacía uso del eslogan "Su dinero puede estar en el lugar equivocado", estrategia que volvió a ser usada en el Mundial del 2014.

Los mensajes virales también permiten conocer las experiencias de terceros con las marcas, beneficiando o generando una crisis para las empresas, tal y como ocurrió con Dave Carroll cuando, después de un vuelo con United Airlines encontró que habían roto su guitarra y al no recibir solución por parte de la empresa decidió subir un video a redes sociales que relata la historia de su instrumento. Dicho video se hizo viral y generó repercusiones negativas en la capitalización de mercado de United Airlines (Berthon, et al., 2012). Incluso, dio lugar a casos de estudio sobre servicio al cliente (Deighton \& Kornfeld, 2010).

La interconexión en redes sociales es, pues, una manera como las empresas pueden conocer las experiencias con sus productos y generar campañas virales mejorando los esfuerzos en mercadeo al aprovechar la estructura de vinculación social, las interacciones entre los usuarios y la influencia social para promover una adopción de decisiones favorables frente a la marca. Estas ventajas se extienden al desarrollo de modelos (i. e. bayesianos) que permitan procesar la data e identificar la probabilidad de ocurrencia para diseñar productos personalizados, basados en la posibilidad de adopción identificada en redes sociales, así como estimar la demanda agregada de la oferta de productos a lo largo del tiempo (Fang, Hu, Li \& Tsai, 2013).

\section{Análisis de datos en las redes sociales}

El contenido en los diferentes tipos de redes sociales guarda una relación con el momento de uso de los usuarios y el posicionamiento de la marca. Así, por ejemplo, YouTube está asociado con la búsqueda de información sobre la marca, mientras que Facebook y Twitter -al ser un vínculo entre los usuarios y la marca- están relacionados con la experiencia, que se transmite por medio de mensajes negativos o positivos; en este sentido, Twitter es percibido como el medio de comunicación más inmediato con los usuarios y Facebook como una autopromoción de experiencias por parte de los usuarios (Smith, Fischer \& Yongjian, 2012).

También se considera que la preferencia por un tipo de red social está relacionada con las características de la personalidad del usuario. Hughes, Rowe, Batey y Lee (2012), quienes tienen en cuenta el modelo de los cinco grandes rasgos de personalidad planteado por Goldberg en 1990, proponen que usuarios con rasgos asociados a la necesidad de cognición y de escrupulosidad prefieren el uso de Twitter, y personas con una predominancia en rasgos de extraversión, sociabilidad y neuroticismo prefieren Facebook.

De la misma manera, la estructura de los mensajes en redes sociales juega un papel importante a la hora de influir en el comportamiento de los usuarios. Es así como el contenido emocional está relacionado con el hecho de que el mensaje sea compartido rápidamente. Según Stieglitz y Dang-Xuan (2013) el contenido positivo está asociado con referentes políticos que son aceptados por los usuarios, mientras el negativo con eventos noticiosos, se convierte en viral. Uno de los medios en el que prevalece la tendencia de identificación de actitudes y 
opiniones es en Twitter, para lo cual aplicaciones como Tweetfeel y Sentiment 140 procesan datos mediante el diseño de algoritmos, clasificando el texto de los mensajes de acuerdo con los sentimientos que se exponen con una exactitud del $85,7 \%$, y eliminando la opción de emociones neutras (Khan, Bashir \& Qamar, 2014).

Según Fischer y Reuber (2011), a partir de la identificación de la orientación de una comunidad y del conjunto de normas al que se adhieren -que se hacen evidentes en la interacción en redes sociales- es las empresas pueden conocer el comportamiento, tendencias y experiencias de consumo de las personas, obteniendo así un evidente beneficio en las estrategias de mercadeo y en las decisiones empresariales. Esto se debe a que se sabe que el tipo de información que es compartida por los usuarios en las redes sociales es un preámbulo de lo que serán afuera de esta (Houghton, et al., 2013).

Investigaciones realizadas en Twitter, como la de Vidal, Ares, Machín y Jaeger (2015), permiten identificar hábitos de consumo en situaciones alimenticias utilizando la clasificación de tuits por palabras. Así, "desayuno", "almuerzo", "cena" y "merienda" sirven para conocer qué se comía, dónde, cuándo y con quién, al igual que la especificación sobre el por qué y la identificación de expresiones emocionales mediante el uso de emoticones, obteniendo como resultado una predominancia en la relación con alimentos como tortas y galletas, o carbohidratos calientes como pasta, arroz y papa; de esta forma también se estableció que el consumo ocurría al día siguiente del tuit.

Asimismo, en un estudio realizado por Houghton et al. (2013), se identificó que las fotografías compartidas por los usuarios en Facebook aluden a la vida personal en contextos familiares, con los amigos y en eventos, mientras que los ejes temáticos son objetos, animales y paisajes, lo cual permite tener una expectativa del tipo de usuario de sus intereses y de la situación en la que comparte la información. La composición de una autofoto (selfie), por su parte, permite entender las características de la persona como su personalidad, estilo de vida y preferencias, las cuales varían acorde a la cultura; sin embargo, el mensaje común de una autofoto es el narcisismo (Senft \& Baym, 2015).

Se ha observado que aspectos como el género de los usuarios influye en el tipo de contenido que, siendo las mujeres las que suelen compartir más información personal en comparación con los hombres (Pan et al., 2004). En este mismo sentido, un estudio realizado por Bamman, Eisenstein y Schnoebelen (2014) pone en evidencia, por ejemplo, que el lenguaje usado por las mujeres contiene abreviaciones y emoticones en un $40 \%$ más que en los hombres, quienes, en un $30 \%$ más que las mujeres, mencionan todo el nombre de la persona que quieren referenciar. También identificaron un $63 \%$ de homofilia entre personas del mismo género, lo cual permite hacer predicciones de atributos personales basados en los atributos sociales.

La forma de relacionarse en línea también cambia en función de la edad, debido a que poblaciones jóvenes valoran la ampliación de la red social, mientras que adultos mayores tienen interacciones emocionales solo con personas cercanas (Oh et al., 2014). Asimismo, se asocian las redes sociales con un público frecuente que pertenece a la generación de los millennials, debido al tipo de interacción, rasgos narcisistas e interés en comunicar constantemente lo que hacen, buscando reflejar una imagen positiva e interesante para sus amigos (Bergman, Fearrington, Davenport \& Bergman, 2011).

De esta manera, las expresiones usadas en redes sociales permiten identificar cambios en el comportamiento, como es el caso de mamás primerizas, quienes presentan conductas diferentes en los tipos de interacciones antes y después del parto. En este caso se evidencia un cambio extremo en el comportamiento en el $71 \%$ frente a la exposición de emociones y vinculación, mientras un $80 \%$ presenta una "ventana de entrenamiento" en los veintiún días antes y después del nacimiento del bebé (De Choudhury, Counts \& Horvitz, 2013).

\section{Estructura web: escalamiento del cerebro}

La WWW no solo traslada las relaciones sociales tradicionales sin conexión a lo en línea, sino que genera interconexiones homólogas a las redes neuronales, conformando así un sistema de conexión inspirado en el sistema nervioso, que permite procesar la información y la conexión entre los usuarios. Al conocer el comportamiento de los usuarios (Nahar, 2012) a partir del uso de modelos probabilísticos de estimación bayesiana que permiten predecir los estilos de comportamiento (Schoen et al., 2013), se potencia la experiencia en línea.

Para la formulación de este tipo de modelos se deben tener en cuenta cuatro aspectos: los procesos de entrada que generan las observaciones de estímulos del entorno, los modelos internos en la toma de decisiones (i. e. las hipótesis), las inferencias que se realizan a partir de las creencias posteriores a las alternativas, y las decisiones que se toman teniendo en cuenta los sistemas de creencias generados después de la decisión (Bitzer, Park, Blankenburg \& Kiebel, 2014). Estos modelos permiten 
conocer, en un periodo de tiempo específico, el porqué del comportamiento de las personas y de los usuarios en línea, por medio de las observaciones sensoriales, el conocimiento del ambiente y las expectativas (Vincent, 2015).

Además, se han diseñado diferentes tipos de sistemas de aprendizaje, por ejemplo, mediante mensajes de texto se logran detectar las emociones como el sarcasmo y la ironía, identificando comportamientos estereotipados a partir de palabras claves, hábitos gramaticales y características en la estructura (Balahur, 2013). De esta mane$\mathrm{ra}$, los modelos bayesianos de inferencia se comportan como un homólogo del sistema sensorial basado en las expectativas y los estímulos sensoriales externos antes de ser procesados cognitivamente. Para poder generar un modelo adaptable al tipo de estímulo externo y prever la posible decisión del usuario (Summerfield \& De Lange, 2014), dichos modelos se basan, principalmente, en las señales visuales.

Por su parte, la formulación de algoritmos permite diseñar máquinas que comprendan el lenguaje y favorezcan una interacción real, para lo cual, a través de la estrategia de filtrar mensajes y convertirse en una red interactiva que va más allá de la socialización tradicional, Facebook hace un primer acercamiento al usuario. Al igual que Oculus, esta red social ha diseñado mecanismos para intensificar la realidad virtual en los mercados masivos (Simonite, 2015). Por tanto, a partir del diseño de sistemas de análisis como algoritmos POS tagging, analizador sintáctico, división binaria o de raíz se pueden entender las características y las opiniones de los usuarios en las diferentes redes sociales (Petz et al., 2014; Petz et al., 2015).

Igualmente, a partir de los correlatos neurológicos y haciendo uso del eye tracking y del FMRI, se puede tener un acercamiento al interés por el estímulo y lo que será la futura conducta de los usuarios. Esta situación se evidenció en una investigación realizada por Djamasbi, Siegel y Tullis (2010), quienes analizaron el interés de los jóvenes por diseños de páginas web que incluyen un mayor número de imágenes y poca información textual; se observó que los sujetos se fijaban en imágenes que tienen rostros, que se interesaban en la búsqueda de logos y que prestaban mayor atención a la información de la compañía ubicada al costado izquierdo de la pantalla. Por su parte, la investigación de Falk, Morelli, Welborn, Dambacher y Lieberman (2013) mostró cómo el efecto Buzz influencia el comportamiento individual y grupal por medio de los mensajes sobre un producto; los resultados indican que en la medida en que se logre difundir acertadamente el mensaje se establece un vínculo emocional con el usuario, lo cual implica una correlación po- sitiva en la activación de la región neuronal y del sistema amigdalar de recompensas, como el estriado ventral.

De esta manera, la evolución de la web se plantea como la integración de los sentidos y de la forma de aprendizaje para interactuar con el usuario, personalizando la experiencia de navegación de acuerdo con a las necesidades de cada uno. Por ejemplo, en investigaciones como la de Kurilovas, Kubilinskiene y Dagiene (2014), y de Lo, Chan y Yeh (2012) se plantean diseños web 3.0 adaptados a los estilos cognitivos de aprendizaje de los estudiantes para consolidar la información. En la medida en que se desarrolla una plataforma más interactiva e interconectada entre los diferentes sistemas y el usuario, en donde el dispositivo es un apoyo que integra, comparte y elabora datos de distintas fuentes y naturaleza, se hace una aproximación al desarrollo de la web semántica, en la cual se crea un universo cognitivo entre los usuarios en el que no solo se intercambia información sino que se le atribuye un significado a esta, implicando una inteligencia colectiva en constante conexión (Lamandini, 2011).

De esta manera, por medio de la web semántica se hace un mapeo y vinculación en la construcción del sentido y de los argumentos, que tiene implicaciones positivas en campos legales y académicos porque facilita la detección de argumentos, al igual que en el análisis de toda la data de la web permitiendo conocer la motivación del usuario y sus esquemas argumentativos (Schneider, Groza \& Passant, 2013). Frente a la vinculación y el acceso a todo tipo de data, al igual que en la generación de redes de aprendizaje, un aspecto a plantearse es el cuidado de la privacidad de los usuarios y de su data (Jeremić, Jovanović \& Gašević, 2013). Consumidores muy expuestos (con poca privacidad) generarían tal cantidad de data que pueden ser fácilmente predecibles, logrando así ser objetivo de la gestión de datos masivos de cara al negocio; sin embargo, la privacidad es un derecho y por tanto existen implicaciones tanto éticas como legales al respecto, afectando así el objetivo predictivo comercial de quienes utilizan estas herramientas (Blattberg, Kim \& Neslin, 2008).

Como resultado de la interacción entre los dispositivos y objetos nace el concepto del "internet de las cosas", por medio del cual se puede hacer procesamiento inteligente de la información para reconocer la identificación de las personas, el tráfico, el flujo de la información en las redes sociales, el monitoreo a gran escala, la predicción y el diagnóstico (Xu, Chen \& Minami, 2012). El internet de las cosas promete mejorar la capacidad de almacenamiento, la fusión de datos y la potencia informática, y generar un diálogo entre los objetos, generando así una mayor interacción con las personas (Qin \& Gu, 2011). Por ende, 
implica una integración entre varias redes tecnológicas, como por ejemplo en el ambiente hospitalario, en el cual, mediante un monitoreo constante de la salud (utilizando sensores y conexiones con cable e inalámbricas), se hace un seguimiento de los objetivos de los pacientes. Asimismo, en la casa y en la oficina se pueden generar espacios más confortables gracias a la graduación de las luces y al monitoreo constante del ambiente; la forma de relacionarse desde la mediación en el entorno social y la actualización automática de la información, también se ven influenciados (Atzori, lera \& Morabito, 2010).

El internet de las cosas, que ha sido considerado como el internet del futuro, busca relacionar el mundo físico con su representación en el sistema de información, brindando así una mayor interconexión, interacción y flujo de datos mediante el uso de sensores y cámaras, para incrementar las experiencias, lo cual abre una oportunidad para ser explotada por las empresas (Haller, Karnouskos \& Schroth, 2008), desde la perspectiva de incrementar la calidad de vida de los usuarios $y$, por ende, generar una experiencia de marca memorable.

\section{Conclusiones}

La evolución de la World Wide Web permite identificar la posibilidad que tienen las empresas para reconocer nuevas oportunidades de mercado y las características de sus clientes, estableciendo el tipo de opiniones y necesidades que refleja el comportamiento en línea de los usuarios, como un preámbulo de lo su comportamiento fuera de la red. De esta manera, al ser los reportes de los usuarios en redes sociales la evidencia de un patrón actitudinal que desemboca en uno comportamental, permiten prever la conducta de dichos usuarios; a esto se suma el factor social, que es entendido como un detonante del contagio de conductas y como un acercamiento a la cognición social. Esta situación favorece las estrategias de mercado, no solo para reconocer opiniones frente a la experiencia con la marca y el producto, sino para identificar clientes potenciales y movilizar comportamientos favorables frente a la marca que están mediados por experiencias positivas y una atención inmediata.

Como consecuencia del reconocimiento de los esquemas y patrones cognitivos de los usuarios se pueden propiciar, mediante el desarrollo web, experiencias cercanas y sensoriales con las marcas que permitan solucionar las necesidades de los clientes, conociéndolos a fondo. Es así como el acceso a una data más detallada y abundante, y gracias a la interconexión entre dispositivos, se puede incrementar la calidad de vida de los usuarios a través del procesamiento inteligente de la información, que permite dar soluciones a distintos problemas, dejan- do un campo abierto por explorar a las empresas. A esto se agrega la forma como las empresas deben cuidar de los clientes y de la privacidad de sus datos, teniendo en cuenta que la exposición de estos genera responsabilidades éticas y legales significativas.

De esta forma, mediante la integración sensorial y el conocimiento del ambiente, sumados a las preferencias de los usuarios identificadas mediante la unificación de data de distinta naturaleza (i.e. información en redes sociales, navegación web), las empresas pueden incrementar la experiencia de marca en línea, lo cual permite fidelizar el comportamiento de los usuarios fuera de esta. Asimismo, es posible atraer futuros posibles clientes, no solo proporcionando un contenido personalizado o previendo las conductas de los usuarios, sino también generando una experiencia multisensorial congruente con la identidad de la marca. Por ejemplo, al navegar por un sitio web los diseños no solo incrementan la observación detallada de imágenes o mensajes impactantes, sino que también permiten que el usuario perciba la impresión de olores asociados con la marca. Otra experiencia multisensorial es la que ocurre, por ejemplo, cuando al momento de comprar algún producto se sobrepasan las barreras de usabilidad web, por ejemplo por medio del uso de gadgets como gafas que permiten hace un viaje $3 \mathrm{D}$ virtual a la tienda, sin la necesidad de desplazarse a esta físicamente, o que al estar en la tienda se entre en un mundo de realidad aumentada, haciendo memorable la experiencia para el cliente.

Igualmente, se observa un campo por explorar en la unificación de conceptos en la web semántica, en donde -mediante la identificación de patrones de búsquedase pueden lograr derivar patrones comportamentales sociales que permitan mejorar la segmentación e identificación de necesidades y la apertura frente a la marca. La web semántica también permitiría identificar los patrones de búsqueda para mejorar la navegación web sugiriendo o recomendando eventos cercanos geográficamente cercanos a los usuarios afines con estos.

Sin embargo, la interconexión y la nueva forma en que se accede a la información generan un desafío para las empresas debido a que el consumo y la compra tradicional también se ven modificadas por la WWW, que puede hacer que las tiendas en línea sean preferidas respecto de las que están fuera de internet, generando así una nueva tendencia de consumo y de acceso a la información, o que la experiencia sin conexión sea aumentada con una interacción en línea. De esta manera, como futuras líneas de investigación, se puede indagar en la forma como la WWW ha modificado las conductas del usuario sin conexión a Internet frente a los estilos de compra, la 
formación de opiniones y la manera como se entiende la realidad, viendo cómo las generaciones más jóvenes cambian el paradigma de usabilidad en línea. También se pueden estudiar las mejores prácticas de realidad virtual, así como el uso y efecto de la realidad aumentada en tiendas sin conexión a Internet. Finalmente, se puede profundizar el entendimiento de la gestión de datos masivos, tanto desde una perspectiva técnica (i. e. desarrollo de nuevos algoritmos), como desde perspectivas sociales (i. e. implicaciones éticas) e incluso legales.

\section{Agradecimientos}

Al Inalde Business School, Universidad de La Sabana, por los recursos aportados para la investigación del área académica de marketing para el grupo de investigación Empresa, Competitividad y Marketing.

\section{Referencias}

Atzori, L., lera, A., \& Morabito, G. (2010). The internet of things: A survey. Computer networks, 54(15), 2787-2805. Balahur, A. (2013). Sentiment analysis in social media texts. In 4th workshop on Computational Approaches to Subjectivity, Sentiment and Social Media Analysis, pp.120-128.

Bamman, D., Eisenstein, J., \& Schnoebelen, T. (2014). Gender identity and lexical variation in social media. Journal of Sociolinguistics, 18(2), 135-160.

Bergman, S. M., Fearrington, M. E., Davenport, S. W., \& Bergman, J. Z. (2011). Millennials, narcissism, and social networking: What narcissists do on social networking sites and why. Personality and Individual Differences, 50(5), 706-711.

Berthon, P. R., Pitt, L. F., Plangger, K., \& Shapiro, D. (2012). Marketing meets Web 2.0, social media, and creative consumers: Implications for international marketing strategy. Business horizons, 55(3), 261-271.

Bitzer, S., Park, H., Blankenburg, F., \& Kiebel, S. J. (2014). Perceptual decision making: drift-diffusion model is equivalent to a Bayesian model. Frontiers in human neuroscience, $8(102), 1-17$

Blattberg, R. C., Kim, BD., \& Neslin, S. A. (2008). Database Marketing - Analizing and Managing Customers. New York, NY (USA): Springer.

Bond, R. M., Fariss, C. J., Jones, J. J., Kramer, A. D., Marlow, C., Settle, J. E., \& Fowler, J. H. (2012). A 61-million-person experiment in social influence and political mobilization.
Nature, 489(7415), 295-298.

Brin, S., \& Page, L. (2012). Reprint of: The anatomy of a large-scale hypertextual web search engine. Computer networks, 56(18), 3825-3833.

Burt, R. S., Kilduff, M., \& Tasselli, S. (2013). Social network analysis: Foundations and frontiers on advantage. Annual review of psychology, 64, 527-547.

Centola, D., \& Macy, M. (2007). Complex contagions and the weakness of long ties1. American journal of Sociology, 113(3), 702-734.

Christakis, N. A., \& Fowler, J. H. (2013). Social contagion theory: examining dynamic social networks and human behavior. Statistics in medicine, 32(4), 556-577.

De Choudhury, M., Counts, S., \& Horvitz, E. (2013). Predicting postpartum changes in emotion and behavior via social media. Proceedings of the SIGCHI Conference on Human Factors in Computing Systems. Abril, pp. 32673276.

Deighton, J., \& Kornfeld, L. (2010). United Breaks Guitars. Harvard Business School Publishing, 1-13.

Djamasbi, S., Siegel, M., \& Tullis, T. (2010). Generation Y, web design, and eye tracking. International Journal of Human-Computer Studies, 68(5), 307-323.

Facebook Newsroom (2013). Feature Phone Milestone: Facebook for Every Phone Reaches 100 Million. Recuperado de: https://newsroom.fb.com/news/2013/07/feature-phone-milestone-facebook-for-every-phone-reaches-100-million/

Facebook Newsroom (2014). A New, Optional Way to Share and Discover Music, TV and Movies. Recuperado de: https://newsroom.fb.com/news/2014/05/a-new-optional-way-to-share-and-discover-music-tv-and-movies/

Falk, E. B., Morelli, S. A., Welborn, B. L., Dambacher, K., \& Lieberman, M. D. (2013). Creating Buzz The Neural Correlates of Effective Message Propagation. Psychological Science, 24(7), 1234-1242.

Fang, X., Hu, P. J. H., Li, Z., \& Tsai, W. (2013). Predicting adoption probabilities in social networks. Information Systems Research, 24(1), 128-145.

Fischer, E., \& Reuber, A. R. (2011). Social interaction via new social media: (How) can interactions on Twitter affect effectual thinking and behavior?. Journal of busi- 
ness venturing, 26(1), 1-18.

Gupta, S., Armstrong, K., \& Clayton, Z. (2011). Social Media. Harvard Business School Press, 1 -14.

Hall, W., \& Tiropanis, T. (2012). Web evolution and Web science. Computer Networks, 56(18), 3859-3865.

Haller, S., Karnouskos, S., \& Schroth, C. (2008). The internet of things in an enterprise context. Vienna: Springer Berlin Heidelberg.

Houghton, D., Joinson, A., Caldwell, N., \& Marder, B. (2013). Tagger's delight? Disclosure and liking in Facebook: the effects of sharing photographs amongst multiple known social circles. Birmingham Business School: Discussion Paper Series.

Hughes, D. J., Rowe, M., Batey, M., \& Lee, A. (2012). A tale of two sites: Twitter vs. Facebook and the personality predictors of social media usage. Computers in Human Behavior, 28(2), 561-569.

Jeremić, Z., Jovanović, J., \& Gašević, D. (2013). Personal learning environments on the social semantic web. Semantic Web, 4(1), 23-51.

Kaplan, A. M., \& Haenlein, M. (2010). Users of the world, unite! The challenges and opportunities of Social Media. Business horizons, 53(1), 59-68.

Khan, F. H., Bashir, S., \& Qamar, U. (2014). TOM:Twitter opinion mining framework using hybrid classification scheme. Decision Support Systems, 57, 245-257.

Kietzmann, J. H., Hermkens, K., McCarthy, I. P., \& Silvestre, B. S. (2011). Social media? Get serious! Understanding the functional building blocks of social media. Business horizons, 54(3), 241-251.

Kurilovas, E., Kubilinskiene, S., \& Dagiene, V. (2014). Web 3.0-Based personalisation of learning objects in virtual learning environments. Computers in Human Behavior, $30,654-662$

Lamandini, A. (2011). Semantic Web. Ricerche di Pedagogia e Didattica. Journal of Theories and Research in Education, 6(1).

Lin, K. Y., \& Lu, H. P. (2011). Why people use social networking sites: An empirical study integrating network externalities and motivation theory. Computers in Human Behavior, 27(3), 1152-1161.
Lo, J. J., Chan, Y. C., \& Yeh, S. W. (2012). Designing an adaptive web-based learning system based on students' cognitive styles identified online. Computers \& Education, 58(1), 209-222.

Michaelidou, N., Siamagka, N. T., \& Christodoulides, G. (2011). Usage, barriers and measurement of social media marketing: An exploratory investigation of small and medium B2B brands. Industrial Marketing Management, 40(7), 1153-1159.

Ministerio de Tecnologías de la Información y las Comunicaciones de Colombia. (2014). 8 de cada 10 colombianos están usando Internet. Recuperado de: http://www. mintic.gov.co/portal/604/w3-article-1629.html

Nahar, K. (2012). Artificial Neural Network. COMPUSOFT, An international journal of advanced computer technology, 1(2) 25-27.

Oh, H. J., Ozkaya, E., \& LaRose, R. (2014). How does online social networking enhance life satisfaction? The relationships among online supportive interaction, affect, perceived social support, sense of community, and life satisfaction. Computers in Human Behavior, 30, 69-78.

Pan, B., Hembrooke, H. A., Gay, G. K., Granka, L. A., Feusner, M. K., \& Newman, J. K. (2004). The determinants of web page viewing behavior: an eye-tracking study. In Proceedings of the 2004 symposium on Eye tracking research \& applications (pp. 147-154).

Petz, G., Karpowicz, M., Fürschuß, H., Auinger, A., Stříteský, V., \& Holzinger, A. (2014). Computational approaches for mining user's opinions on the Web 2.0. Information Processing \& Management, 50(6), 899-908.

Petz, G., Karpowicz, M., Fürschuß, H., Auinger, A., Stř́iteský, V., \& Holzinger, A. (2015). Reprint of: Computational approaches for mining user's opinions on the Web 2.0. Information Processing \& Management, 51(4), 510-519.

Qin, X., \& Gu, Y. (2011). Data fusion in the Internet of Things. Procedia Engineering, 15, 3023-3026.

Rahwan, l., Zablith, F., \& Reed, C. (2007). Laying the foundations for a world wide argument web. Artificial intelligence, 171(10), 897-921.

Rodríguez, D. (2013). Memecracia: Los virales que nos gobiernan. Cómo las ideas contagiosas usan internet para manipular tu mente. Barcelona: Grupo Planeta Spain.

Schneider, J., Groza, T., \& Passant, A. (2013). A review of ar- 
gumentation for the social semantic web. Semantic Web, 4(2), 159-218.

Schoen, H., Gayo-Avello, D., Takis Metaxas, P., Mustafaraj, E., Strohmaier, M., \& Gloor, P. (2013). The power of prediction with social media. Internet Research, 23(5), 528-543. Sen, R. (2005). Optimal search engine marketing strategy. International Journal of Electronic Commerce, 10(1), 9-25.

Senft, T. M., \& Baym, N. K. (2015). Selfies Introduction What Does the Selfie Say? Investigating a Global Phenomenon. International Journal of Communication, 9, (19), 1588- 1606.

Simonite, T. (2015). Teaching Machines to Understand Us, MIT Technology Review. Recuperado de https:// www.technologyreview.com/s/540001/teaching-machines-to-understand-us/

Smith, A. N., Fischer, E., \& Yongjian, C. (2012). How does brand-related user-generated content differ across YouTube, Facebook, and Twitter? Journal of Interactive Marketing, 26(2), 102-113.

Smith, J. R., Terry, D. J., Manstead, A. S., Louis, W. R., Kotterman, D., \& Wolfs, J. (2008). The attitude-behavior relationship in consumer conduct: The role of norms, past behavior, and self-identity. The Journal of Social Psychology, 148(3), 311-334.

Stieglitz, S., \& Dang-Xuan, L. (2013). Emotions and information diffusion in social media-sentiment of microblogs and sharing behavior. Journal of Management Information Systems, 29(4), 217-248.

Summerfield, C., \& de Lange, F. P. (2014). Expectation in perceptual decision making: neural and computational mechanisms. Nature Reviews Neuroscience,15(11), 745756.
Think with google, $(2015$, a). 4 New Moments Every Marketer Should Know. Recuperado de: https://think.storage.googleapis.com/docs/4-new-moments-every-marketer-should-know.pdf

Think with google $(2015$, b). Cooking Trends Among Millennials: Welcome to the Digital Kitchen. Recuperado de: https://www.thinkwithgoogle.com/articles/cooking-trends-among-millennials.html

Vidal, L., Ares, G., Machín, L., \& Jaeger, S. R. (2015). Using Twitter data for food-related consumer research: A case study on "what people say when tweeting about different eating situations". Food Quality and Preference, 45, 58-69.

Vincent, B. T. (2015). Bayesian accounts of covert selective attention: A tutorial review. Attention, Perception, \& Psychophysics, 77(4), 1013-1032.

Wilson, R. F., \& Pettijohn, J. B. (2008). Search engine optimisation: A primer on outsourcing key tasks. Journal of Direct, Data and Digital Marketing Practice, 10(2), 133149.

Xu, X., Chen, T., \& Minami, M. (2012). Intelligent fault prediction system based on internet of things. Computers \& Mathematics with Applications, 64(5), 833-839.

Yang, S., \& Ghose, A. (2010). Analyzing the relationship between organic and sponsored search advertising: Positive, negative, or zero interdependence? Marketing Science, 29(4), 602-623.

Zuze, H., \& Weideman, M. (2013). Keyword stuffing and the big three search engines. Online Information Review, $37(2), 268-286$. 